\title{
Development and Ground Evaluation of Ground-Target Tracking Control of Microsatellite RISESAT
}

\author{
By Shinya Fujita, Yuji Sato, Toshinori Kuwahara, Yuji SAKAmoto, and Kazuya Yoshida \\ Department of Aerospace Engineering, Tohoku University, Sendai, Japan
}

(Received June 23rd, 2017)

\begin{abstract}
Space Robotics Laboratory (SRL) of Tohoku University is currently developing a 50-kg-class Earth observation satellite "RISESAT". In this paper, we propose a new ground-target tracking control algorithm and attitude control system for RISESAT. The primal mission of RISESAT is multi-spectral observation of the Earth surface using a 5 m GSD HighPrecision-Telescope (HPT) with liquid crystal tunable filters. During an observation, the HPT takes a dozen images of a ground target by changing observation bands. General Earth observation satellites using push-broom cameras require high attitude stability to obtain continuous images. However, RISESAT has to track a ground target with an accuracy of $0.1^{\circ}$ and $0.008 \%$ s attitude stability because imaging and wavelength switching take few seconds in total. Therefore, we propose a new ground-target tracking algorithm which can keep orientation of ground-target image to a constant direction which satellite operator requires. Evaluations of the algorithms were carried out by hardware-in-the-loop simulator "MEVI $\mu \mathrm{S}$ " which is a satellite system verification environment developed by SRL. We confirm that the algorithm satisfies the mission requirements under the influence of sensor noise and computation time limits.
\end{abstract}

Key Words: Attitude Control, Ground-Target Tracking, Hardware-in-the-Loop Simulation, Microsatellite, Multi-Spectral Observation

\section{Nomenclature}

$\begin{array}{lll}\boldsymbol{C} & : & \text { direct cosine matrix } \\ \boldsymbol{e} & : & \text { basis vector } \\ h & : & \text { angular momentum } \\ J & : & \text { inertia tensor } \\ \boldsymbol{k} & : & \text { control gain } \\ \boldsymbol{q} & : & \text { quaternion } \\ \boldsymbol{R} & : & \text { geocentric ground target position vector } \\ \boldsymbol{r} & : & \text { geocentric satellite position vector } \\ \boldsymbol{T} & : & \text { torque } \\ t & : & \text { time } \\ \Delta t & : & \text { time step size } \\ \boldsymbol{u} & : & \text { unit vector } \\ \theta & : & \text { rotation angle around boresight vector } \\ \lambda & : & \text { longitude } \\ \varphi & : & \text { geodetic latitude } \\ \boldsymbol{\omega} & : & \text { angular velocity }\end{array}$

Subscripts

$b \quad$ : body-fixed system

$d$ : desired value

$e \quad$ : error value

$f \quad$ : geodetic coordinates system

$i \quad$ : inertial system

$N \quad$ : north direction

$n \quad:$ step number in discrete-time system

$w$ : wheel

$x \quad: \quad 1$ st axis of orthogonal coordinates system

$y \quad:$ 2nd axis of orthogonal coordinates system

$z \quad$ : 3rd axis of orthogonal coordinates system

\section{Introduction}

\subsection{Mission of RISESAT}

Space Robotics Laboratory (SRL) of Tohoku University is currently developing a series of 50-kg-class microsatellites for Earth observation. RISESAT (Rapid International Scientific Experiment Satellite) (Fig. 1) which is our 4th microsatellite has been selected to "Innovative Satellite Technology Demonstration Program" provided by Japan Aerospace Exploration Agency (JAXA) and planned to be launched by the Epsilon Rocket in Japanese fiscal year of 2017.

The primal mission of RISESAT is high-resolution multispectral observation of the Earth surface. Multi-spectral observation images are useful to obtain environmental information such as vegetation, growing conditions of farms and mineral resources, so recent 50 - $100 \mathrm{~kg}$ class microsatellites dedicated for optical remote sensing missions have equipment which can observe 4 bands (Red + Green + Blue + Near Infrared. ${ }^{1,2)}$ However, dozens of observation bands are required to meet the demands from fields of agriculture, forestry, fisheries and mining industries. ${ }^{3)}$

Therefore, Liquid Crystal Tunable Filters (LCTF) was developed by Hokkaido University. ${ }^{4}$ The LCTF is a small spectroscope which can electrically select an observation band

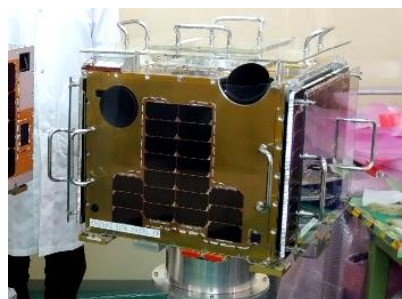

Fig. 1. RISESAT.

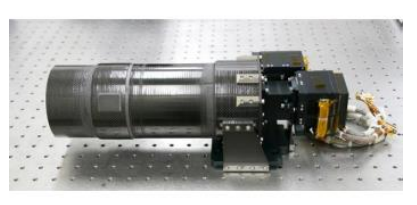

Fig. 2. HPT 
from 680 bands in 420 - $1050 \mathrm{~nm}$. With combination of 1000 $\mathrm{mm}$ focal length cassegrain telescope and the LCTF, HighPrecision Telescope (HPT) was developed for RISESAT. Specifications of RISESAT and HPT are shown in Table 1 and Table 2 respectively.

Table 1. Specifications of RISESAT.

\begin{tabular}{|c|c|c|c|c|}
\hline \multirow{2}{*}{$\frac{\text { Items }}{\text { Dimensions }}$} & & \multicolumn{3}{|l|}{ Values } \\
\hline & & $500 \times 500 \times 50$ & $\mathrm{~mm}$ & \\
\hline Weight & & $55 \mathrm{~kg}$ & & \\
\hline Inertia Tensor & & {$\left[\begin{array}{cc}1.70 & -0.03 \\
-0.03 & 1.69 \\
-0.03 & 0.02\end{array}\right.$} & $\left.\begin{array}{c}-0.01 \\
0.02 \\
1.88\end{array}\right]$ & $\mathrm{kgm}^{2}$ \\
\hline Orbit & $\begin{array}{l}\text { type } \\
\text { altitude }\end{array}$ & $\begin{array}{l}\text { Sun Synchronc } \\
500-700 \mathrm{~km}\end{array}$ & & \\
\hline Power & $\begin{array}{l}\text { Max. generation } \\
\text { Max. consumption }\end{array}$ & $\begin{array}{l}100 \mathrm{~W} \\
50 \mathrm{~W}\end{array}$ & & \\
\hline
\end{tabular}

Table 2. Specifications of HPT.

\begin{tabular}{ll}
\hline Items & Values \\
\hline Observation band & 680 bands $(420-1050 \mathrm{~nm})$ \\
Ground resolution & $5 \mathrm{~m}$ \\
Field of view & $0.2^{\circ}$ \\
Telescope type & Cassegrain \\
Diameter & $100 \mathrm{~mm}$ \\
Focal length & $1000 \mathrm{~mm}$ \\
\hline
\end{tabular}

A problem of multi-spectral observation is a long exposure time compared to panchromatic observation. Ground resolution of conventional multi-spectral observation using push-bloom scanner is relatively low (approx. 30m GSD) because orbital motion of the satellite interferes image acquisition. ${ }^{5)}$

Therefore, RISESAT applies a new observation method named "Multi-spectral Snapshot Observation". In this method, a two-dimension CCD sensor with LCTF takes dozens of images of ground target by changing observation bands. To keep the target in the field of view and avoid blurring, the satellite tracks the ground target precisely by maneuvering entire satellite body. RISESAT has to track a ground target with an accuracy of $0.1^{\circ}$ and $0.008^{\circ} / \mathrm{s}$ attitude stability because imaging and observation band switching take few seconds in total.

In this paper, we propose a precision ground-target tracking control algorithm dedicated for multi-spectral snapshot observation.

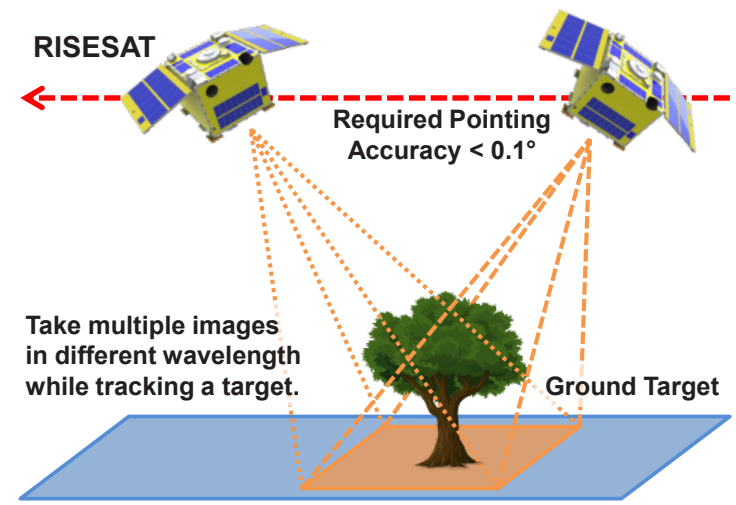

Fig. 3. Idea of multi-spectral snapshot observation.

\subsection{Relation to prior work}

Several attitude control algorithms for ground-target tracking are proposed in prior works.

Goeree et al. proposed a ground-station tracking algorithm for laser communication for 70-kg-class microsatellite "UASat" developed by University of Arizona. Their simulation results showed that tracking accuracy is smaller than $0.005^{\circ}$ under a condition of $0.25 \mathrm{~s}$ sampling time, and errorless attitude determination. However, this algorithm is not appropriate for snapshot observation since angle around optical axis is not included. ${ }^{7)}$

Chen et al. proposed a proposed a ground-target tracking algorithm for Earth observation. However, this algorithm has a problem that satellite cannot satisfy desired attitude and desired angular velocity simultaneously since desired angular velocity is derived from geometrical condition which is unrelated to desired attitude. ${ }^{8)}$

Based on the above, Kondo et al. proposed a ground-target tracking algorithm for Earth observation, and its desired attitude and angular velocity are consistent. In this algorithm, they derived desired attitude using Euler axis rotation between an optical equipment and direction toward ground target. Therefore, orientation of ground-target image cannot be fixed and continuously rotating along optical axis during observation. ${ }^{9)}$

An advantage of our new algorithm is attitude control around optical axis. Our ground-target tracking algorithm can keep orientation of image in the field of view. This characteristic makes synthesis of images taken by different bands easier. To verify the algorithm, we carried out hardware-in-the-loop simulations. This evaluation method grantee accuracy of our control law in practical environment under the influence of attitude/orbit determination error and calculation delay.

\section{Attitude Control System of RISESAT}

In this chapter, attitude control equipment related to groundtarget tracking control are shown.

\subsection{Attitude control unit (ACU)}

Attitude Control Unit (ACU) is a Field-Programmable Gate Array (FPGA) based computer, and OpenRISC1200 32bit softcore processor developed by OpenCore.org is applied. Parallel processing capability of FPGA enables to communicate 12 attitude sensors and actuators simultaneously. Commands and telemetry data are exchanged through Satellite Central Unit (SCU). All sensors, actuators and computers are

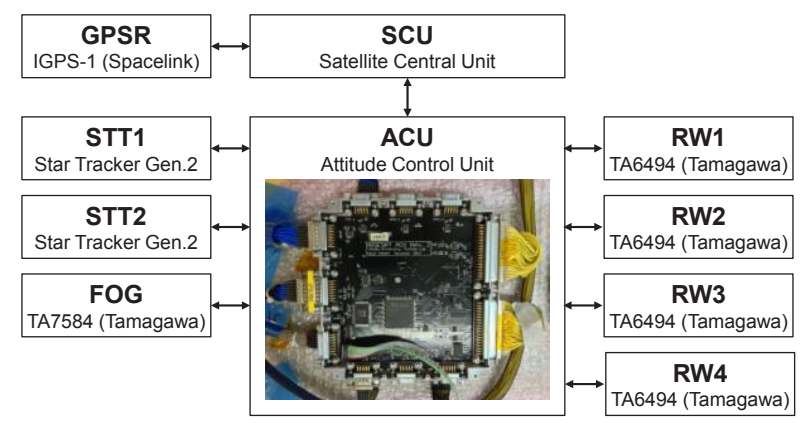

Fig. 4. Diagram of attitude control system. 
already verified on orbit by $50 \mathrm{~kg}$-class microsatellite "DIWATA-1". ${ }^{10)}$ Diagram of attitude control system is shown in .

\subsection{Attitude sensors \\ Star trackers (STTs)}

Two star trackers developed in cooperative research of Meisei electric co., 1td. and SRL are installed. Although STTs are most accurate attitude sensor, they cannot determine its attitude when angular velocity exceed $0.1 \%$ s. Specifications are shown in Table 3.

Table 3. Specifications of star trackers.

\begin{tabular}{|c|c|}
\hline Items & Values \\
\hline Field of view & $27.8^{\circ}$ \\
\hline Data update & $1 \mathrm{~Hz}$ \\
\hline Maximum angular velocity & $0.1 \% \mathrm{~s}$ \\
\hline Accuracy $(3 \sigma) \quad$ around camera axis & $0.6^{\circ}$ \\
\hline other axes & $0.06^{\circ}$ \\
\hline
\end{tabular}

\section{Fiber optical gyroscope (FOG)}

RISESAT is equipped with a fiber optical gyroscope TA7584 developed by Tamagawa seiki co., 1td. Specifications are shown in Table 4.

Table 4. Specifications of a fiber optical gyroscope.

\begin{tabular}{ll}
\hline Items & Values \\
\hline Detection range & $\pm 5 \% \mathrm{~s}$ \\
Data update & $20 \mathrm{~Hz}$ \\
Allan variance (at $1 \mathrm{~s})$ & $1.1 \% \mathrm{~h}$ \\
Random noise $(1 \sigma)$ & $0.00124^{\circ} / \mathrm{s}$ \\
\hline
\end{tabular}

\section{GPS receiver (GPSR)}

GPS receiver IGPS-1 developed by Spacelink Co., Ltd is used to obtain positioning information. Latitude, longitude and altitude are shown in WGS84 coordinates system and time is expressed by Coordinated Universal Time (UTC). Pulse Per Second (PPS) signal generated by GPSR is used not only attitude control system but also synchronizing entire satellite system. Specifications are shown in Table 5.

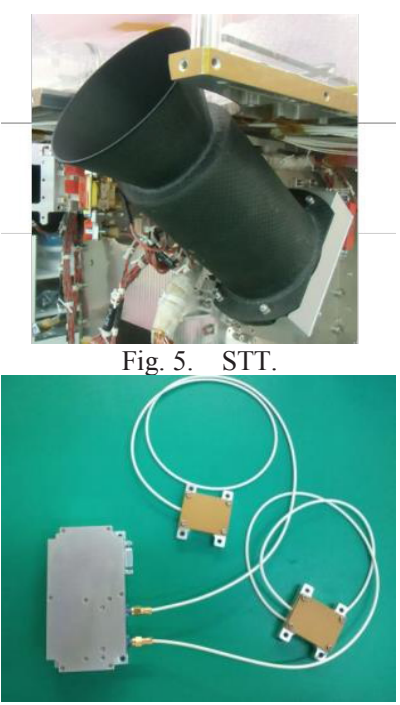

Fig. 7. GPSR.

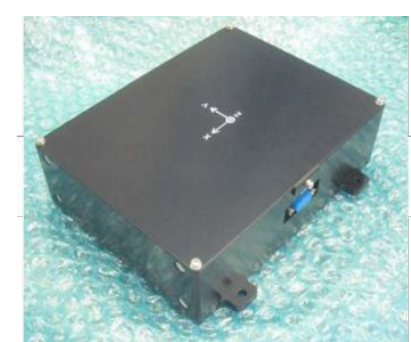

Fig. 6. FOG.

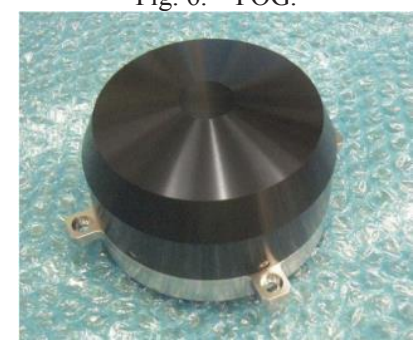

Fig. 8. RW.

\begin{tabular}{ll} 
Table 5. & Specifications of a GPS receiver. \\
\hline Items & Values \\
\hline Coordinate system & WGS84 \\
Receiver type & $\mathrm{L} 1 \mathrm{C} / \mathrm{A}$ \\
Data update & $1 \mathrm{~Hz}$ \\
Position Accuracy (RMS) & $17 \mathrm{~m}$ \\
\hline
\end{tabular}

\subsection{Actuators}

\section{Reaction wheels (RWs)}

RISESAT is equipped with 4 reaction wheels TA6494 developed by Tamagawa seiki co., ltd. RWs are mounted by 4 skew alignment. Specifications are shown in Table 6.

\begin{tabular}{|c|c|}
\hline Items & Values \\
\hline Power consumption (at $3000 \mathrm{rpm}$ ) & $2.2 \mathrm{~W}$ \\
\hline Maximum angular momentum & $0.3 \mathrm{Nms}$ \\
\hline Maximum speed & $\pm 4000 \mathrm{rpm}$ \\
\hline Torque scale factor & $0.03 \mathrm{Nm} / \mathrm{A}$ \\
\hline Data update & $20 \mathrm{~Hz}$ \\
\hline
\end{tabular}

\section{Ground-Target Tracking Control Algorithm}

In this chapter, we propose derivation of a new algorithm to calculate desired attitude, angular velocity and torque for ground-target tracking control.

\subsection{Definitions of coordinates system}

We define several coordinates systems used in this paper as follows:

- Geocentric Celestial Reference System (GCRS)

GCRS is an Earth centered inertial coordinates system. The $x$-axis points towards the $\mathrm{J} 2000.0$ mean equinox. The $z$-axis points to the celestial pole, and the $y$-axis is defined to form a right-handed orthogonal reference system. ${ }^{11)}$

- International Terrestrial Reference System (ITRS)

ITRS is an Earth centered world geodetic coordinates system and maintained by International Earth Rotation and Reference System Service (IERS). The $x$-axis points towards a node of prime meridian and equator. The $z$-axis points to the celestial pole, and the $y$-axis is defined to form a right-handed orthogonal reference system. WGS84 coordinates used in GPSR can be dealt as same coordinates system practically. ${ }^{11)}$

- Horizontal Coordinate System (HCS)

HCS is a local tangent coordinates system. The origin is defined at a given point on Earth. The $x$-axis points towards east direction. The $y$-axis points towards north direction, and the $z$-axis points towards zenith direction. ${ }^{2)}$

- Body-fixed Coordinate System (BCS)

$\mathrm{BCS}$ is a coordinates system originate from satellite center of mass. The axes are defined as .

- Target-tracking Coordinate System (TCS)

TCS is a coordinates system for ground-target tracking. Definitions and derivation will be detailed later.

\subsection{Derivation of ground-target tracking attitude}

First, we calculate desired boresight vector which is a vector points towards satellite center of mass to ground target in 
inertial system. When a ground-target location $\boldsymbol{R}_{\text {ITRS }} \in \mathbb{R}^{3}$ is given in geodetic coordinate system ITRS, a ground-target location $\boldsymbol{R}_{G C R S} \in \mathbb{R}^{3}$ in inertial coordinates system GCRS can be expressed as (1). P, N, R, W $\in S O(3)$ are coordinates transformation matrix which represent precession, nutation, sidereal-rotation and polar-motion respectively. ${ }^{11)}$

$$
\boldsymbol{R}_{G C R S}=[\boldsymbol{P}(t)][\boldsymbol{N}(t)][\boldsymbol{R}(t)][\boldsymbol{W}(t)] \boldsymbol{R}_{I T R S}
$$

When we assume that a current position of satellite $\boldsymbol{r}_{\text {GCRS }} \in$ $\mathbb{R}^{3}$ is known in GCRS, boresight vector $\boldsymbol{x}_{\text {bore }} \in \mathbb{R}^{3}$ will be calculated as:

$$
\boldsymbol{x}_{\text {bore }}=\boldsymbol{R}_{G C R S}-\boldsymbol{r}_{G C R S}
$$

Next, we consider rotation angle around optical axis of observation equipment. In this algorithm, orientation of ground-target image in the field of view can be fixed. As a reference of ground-target orientation, we use unit north direction vector $\boldsymbol{u}_{N f} \in \boldsymbol{S}^{2}$ in ITRS. Since $\boldsymbol{u}_{N f}$ is equivalent to basis vector of HCS, it can be calculated as Eq. (3). ${ }^{12)}$ In GCRS, unit north direction vector $\boldsymbol{u}_{N i} \in \boldsymbol{S}^{2}$ is expressed as (4) using same transformation matrix in Eq. (1). $\varphi$ and $\lambda$ are longitude and geodetic latitude of ground target respectively.

$$
\begin{gathered}
\boldsymbol{u}_{N f}=\left[\begin{array}{c}
-\sin \varphi \cos \lambda \\
-\sin \varphi \sin \lambda \\
\cos \varphi
\end{array}\right] \\
\boldsymbol{u}_{N i}=[\boldsymbol{P}(t)][\boldsymbol{N}(t)][\boldsymbol{R}(t)][\boldsymbol{W}(t)] \boldsymbol{u}_{N f}
\end{gathered}
$$

Target-tracking Coordinate System (TCS) can be created with $\boldsymbol{x}_{\text {bore }}$ and $\boldsymbol{u}_{N i}$. The $z$-axis points along desired boresight vector. The $y$-axis corresponds to orthogonal projection of $\boldsymbol{u}_{N i}$ to perpendicular plane of the $z$-axis. The $x$-axis is defined to form a right-handed orthogonal reference system.

The z-axis of TCS $\boldsymbol{e}_{z} \in \boldsymbol{S}^{2}$ is equivalent to a unit vector of desired boresight vector $\boldsymbol{x}_{\text {bore }}$ given by

$$
\boldsymbol{e}_{z}=\boldsymbol{u}_{\text {bore }}=\frac{\boldsymbol{x}_{\text {bore }}}{\left\|\boldsymbol{x}_{\text {bore }}\right\|}
$$

Since $y$-axis of TCS $\boldsymbol{e}_{y} \in \boldsymbol{S}^{2}$ corresponds to orthogonal projection of $\boldsymbol{u}_{N i}$ to perpendicular plane of the $z$-axis, the $x$ axis of TCS $\boldsymbol{e}_{x} \in S^{2}$ will be calculated as:

$$
\boldsymbol{e}_{x}=\frac{\boldsymbol{u}_{N i} \times \boldsymbol{e}_{z}}{\left\|\boldsymbol{u}_{N i} \times \boldsymbol{e}_{Z}\right\|}
$$

Remaining $y$-axis can be derived from cross products of the $x$ and $z$-axis. Therefore, direct cosine matrix which represent TCS $\boldsymbol{C}_{T C S} \in S O(3)$ can be expressed as:

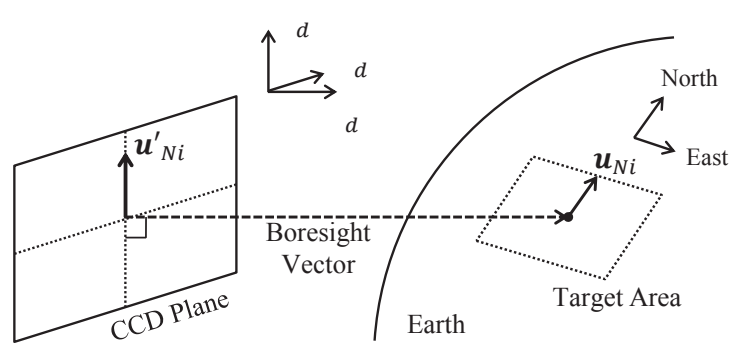

Fig. 9. Definition of target-tracking coordinate system (TCS).

$$
\begin{gathered}
\boldsymbol{e}_{y}=\frac{\boldsymbol{e}_{z} \times \boldsymbol{e}_{x}}{\left\|\boldsymbol{e}_{z} \times \boldsymbol{e}_{x}\right\|} \\
\boldsymbol{C}_{T C S}=\left[\begin{array}{lll}
\boldsymbol{e}_{x} & \boldsymbol{e}_{y} & \boldsymbol{e}_{z}
\end{array}\right]^{T}
\end{gathered}
$$

Orientation $\theta$ of target image to north direction can be modified by direct cosine matrix $\boldsymbol{C}_{\theta} \in S O(3)$ which is a coordinates transformation matrix around the $z$-axis. Desired attitude for ground-target tracking $\boldsymbol{C}_{d} \in S O(3)$ will be expressed as:

$$
\begin{gathered}
\boldsymbol{C}_{d}=\boldsymbol{C}_{\theta} \boldsymbol{C}_{T C S} \\
\boldsymbol{C}_{\theta}=\left[\begin{array}{ccc}
\cos \theta & \sin \theta & 0 \\
-\sin \theta & \cos \theta & 0 \\
0 & 0 & 1
\end{array}\right]
\end{gathered}
$$

Following the prior work proposed by Kondo et al., desired angular velocity can be determined as time derivative of desired attitude. The attitude kinematic equation using quaternion representation can be described by Eqs. (10) and (11) generally.

$$
\begin{aligned}
& \dot{\boldsymbol{q}}=\frac{1}{2} \boldsymbol{\Omega} \boldsymbol{q} \\
& \left.\boldsymbol{\Omega}=\begin{array}{cccc}
0 & \omega_{z} & -\omega_{y} & \omega_{x} \\
-\omega_{z} & 0 & \omega_{x} & \omega_{y} \\
\omega_{y} & -\omega_{x} & 0 & \omega_{z} \\
-\omega_{x} & -\omega_{y} & -\omega_{z} & 0
\end{array}\right]
\end{aligned}
$$

Assuming a time derivative of desired attitude quaternion $\dot{\boldsymbol{q}}_{d}$ is given, desired angular velocity vector $\boldsymbol{\omega}_{d} \in \mathbb{R}^{3}$ can be calculated as:

$$
\boldsymbol{\Omega}_{d}=2 \dot{\boldsymbol{q}}_{d} \boldsymbol{q}_{d}^{-1}
$$

\subsection{Quaternion-based controller}

To determine desired control torque, we apply quaternionbased rate/attitude tracking control algorithms (Eq. (13)) proposed by Weiss. ${ }^{13)} \boldsymbol{\omega}_{b}$ is angular velocity of a satellite body. $\boldsymbol{k}_{D}$ and $\boldsymbol{k}_{P}$ are positive derivative and positive proportional gain vector respectively. $\boldsymbol{h}_{w}$ represent angular momentum of reaction wheels.

Although the quaternion feedback regulator ${ }^{14)}$ (Eq. (14)) proposed by Wie et al. is also well known as a quaternion-based controller however, feedforward term included in Weiss's algorithm is indispensable to achieve agile attitude maneuver for the ground-target tracking control.

$$
\boldsymbol{T}_{d}=-\boldsymbol{k}_{D} \boldsymbol{\omega}_{\boldsymbol{e}}-\boldsymbol{k}_{P} \boldsymbol{q}_{e}-\boldsymbol{\omega}_{b} \times\left(\boldsymbol{J} \boldsymbol{\omega}_{b}+\boldsymbol{h}_{w}\right)+J \dot{\boldsymbol{\omega}}_{d}
$$

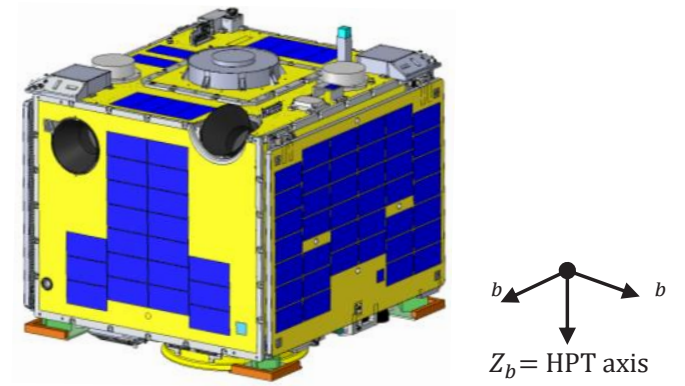

Fig. 10. Definition of body-fixed coordinate system (BCS). 


$$
\begin{gathered}
\omega_{e}=\omega_{b}-\omega_{d} \\
\boldsymbol{q}_{e}=\boldsymbol{q}_{d}^{-1} \boldsymbol{q} \\
\boldsymbol{T}_{d}=-\boldsymbol{k}_{D} \boldsymbol{\omega}_{e}-\boldsymbol{k}_{P} \boldsymbol{q}_{e}-\boldsymbol{\omega} \times\left(\boldsymbol{J} \boldsymbol{\omega}+\boldsymbol{h}_{w}\right)
\end{gathered}
$$

\section{Implementation of the Algorithm for RISESAT}

In this chapter, we explain practical a way to implement the ground-target tracking algorithm to the attitude control system of RISESAT.

\subsection{Derivation of desired attitude \\ Transformation between ITRS and GCRS}

To derive desired attitude, transformation matrices between geodetic coordinates ITRS and inertial coordinates GCRS are required because both satellite positioning information by GPSR and a ground target location are given in ITRS. RISESAT adopt IAU76/FK5-based Earth's rotation models shown in Table 7. Although accuracy of these models is inferior to latest CIO-based IAU2000/2006 models, they contribute to shorten calculation time. ${ }^{11,15)}$ Due to the limitation of computation resource, order of the nutation model is reduced to 10th from original 109th.

When calculating these transformation matrices, not only UTC coming from GPSR but also Universal Time 1 (UT1) and Terrestrial Time (TT) are required. The time correction parameter DUT1 determined by IERS will be sent from ground stations every day since UT1 is a time standard which is affected by fluctuations of Earth's rotation. ${ }^{16)}$

Table 7. Transformation between ITRS and GCRS

\begin{tabular}{lll}
\hline Model & & Calculation time \\
\hline Precession & IAU 1976 & $20 \mathrm{~ms}$ \\
Nutation & IAU 1980 (10th order) & $90 \mathrm{~ms}$ \\
Sidereal-rotation & IAU 1982 & $20 \mathrm{~ms}$ \\
Polar-motion & IERS 1996 & $10 \mathrm{~ms}$ \\
\hline
\end{tabular}

\section{Time derivative of quaternion and angular velocity}

In order to compute time derivative of desired attitude quaternion $\left.\dot{\boldsymbol{q}}_{d}\right|_{n}$, we use the backward Euler method. In discrete-time system, $\left.\dot{\boldsymbol{q}}_{d}\right|_{n}$ can be expressed as Eq. (15). Desired angular acceleration $\left.\dot{\boldsymbol{\omega}}_{d}\right|_{n}$ can be also derived as Eq. (16). $\Delta t$ represent time step size in discrete-time system, and $\Delta t=1 \mathrm{~s}$ in RISESAT ACU.

$$
\begin{gathered}
\left.\dot{\boldsymbol{q}}_{d}\right|_{n}=\frac{\left.\left.\boldsymbol{q}_{d}^{-1}\right|_{n-1} \boldsymbol{q}_{d}\right|_{n}}{\Delta t} \\
\left.\dot{\boldsymbol{\omega}}_{d}\right|_{n}=\frac{\left.\boldsymbol{\omega}_{d}\right|_{n}-\left.\boldsymbol{\omega}_{d}\right|_{n-1}}{\Delta t}
\end{gathered}
$$

\subsection{Attitude determination}

\section{Infinite impulse response filter for a star tracker}

Attitude filtering method for star trackers is required since random noise in sensor output exceeds desired attitude control accuracy $0.1^{\circ}$. Although Extended Kalman Filters are widely used in precision attitude determination by star trackers and gyroscopes, ${ }^{17)}$ RISESAT ACU which does not have floating point unit cannot execute a EKF within $1 \mathrm{~s}$ control cycle. Therefore, we developed an Infinite Impulse Response (IIR) filter using a star tracker and a gyroscope.

Attitude quaternion after filtering $\left.\boldsymbol{q}\right|_{n}$ can be obtained by Eq. (17). Equation (17) is an equation which derive average of two quaternions, ${ }^{18)}$ and a coefficient $w$ works as a weight of feedbacked quaternion $\left.\widehat{\boldsymbol{q}}\right|_{n}$ against latest observation quaternion $\left.\left.\boldsymbol{q}_{S T T}\right|_{n} \cdot \widehat{\boldsymbol{q}}\right|_{n}$ can be calculated by quaternion kinematics using attitude quaternion $\left.\boldsymbol{q}\right|_{n-1}$ determined in previous step and skew-symmetric matrix of current satellite angular velocity $\left.\boldsymbol{\Omega}\right|_{n}$ measured by gyroscope.

$$
\begin{gathered}
\left.\boldsymbol{q}\right|_{n}= \pm \frac{\left.(1-w+z) \widehat{\boldsymbol{q}}\right|_{n}+\left.2 w\left(\left.\left.\widehat{\boldsymbol{q}}\right|_{n}{ }^{T} \boldsymbol{q}_{S T T}\right|_{n}\right) \boldsymbol{q}_{S T T}\right|_{n}}{\left\|\left.(1-w+z) \widehat{\boldsymbol{q}}\right|_{n}+\left.2 w\left(\left.\left.\widehat{\boldsymbol{q}}\right|_{n}{ }^{T} \boldsymbol{q}_{S T T}\right|_{n}\right) \boldsymbol{q}_{S T T}\right|_{n}\right\|} \\
z=\sqrt{(1-w)^{2}+4 w\left(\left.\left.\widehat{\boldsymbol{q}}\right|_{n}{ }^{T} \boldsymbol{q}_{S T T}\right|_{n}\right)^{2}} \\
\left.\widehat{\boldsymbol{q}}\right|_{n}=\frac{\left.\boldsymbol{q}\right|_{n-1}+\left.\left.\frac{\Delta t}{2} \boldsymbol{\Omega}\right|_{n} \boldsymbol{q}\right|_{n-1}}{\left\|\left.\boldsymbol{q}\right|_{n-1}+\left.\left.\frac{\Delta t}{2} \boldsymbol{\Omega}\right|_{n} \boldsymbol{q}\right|_{n-1}\right\|}
\end{gathered}
$$

\section{Angular velocity integration}

STTs cannot determine its attitude when angular velocity of the satellite exceed $0.1 \%$ s. However, maximum angular velocity during tracking maneuver amounts to $0.8 \%$ shen flying over the target. Therefore, angular velocity integration method (18) is utilized to obtain precision attitude continuously. In this calculation, $\Delta t=0.1 \mathrm{~s}$.

\section{Evaluation}

\subsection{Hardware-in-the-loop simulator}

Evaluation of algorithm and attitude control equipment were carried out by a "MEVI $\mu$ S" (Model based Evaluation and Verification for Integration of $\mu$-Satellite) which is a hardwarein-the-loop simulation and verification environment developed by SRL. ${ }^{19)}$ For this evaluation, real engineering model of RISESAT bus system except for attitude sensors and actuators were integrated. Mathematical models of attitude sensors and

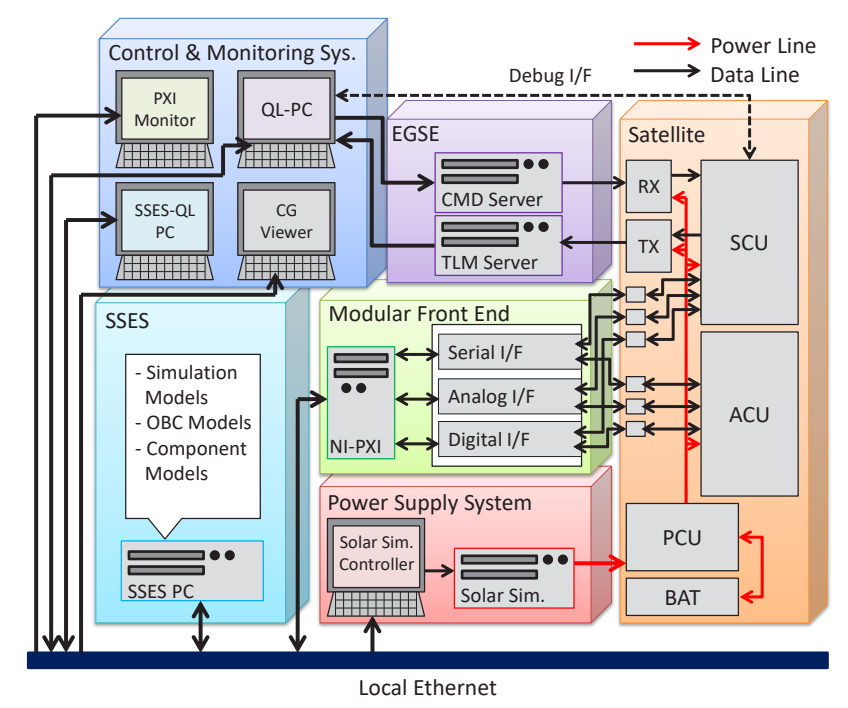

Fig. 11. Diagram of MEVI $\mu$ S system. 
actuators are created using on-orbit evaluation results by DIWATA-1 or on-ground hardware evaluation results.

\subsection{Simulation conditions}

Simulation was carried out from January 1st 2016 00:00:00 (UTC) for 30 minutes using a Two-Line Elements of HODOYOSHI-1 which resembles to the orbit RISESAT. ${ }^{20)} \mathrm{A}$ ground-target location was set at $75.0097^{\circ} \mathrm{S}, 10.25444^{\circ} \mathrm{E}$ which is a sub-satellite point at 00:15:00. The simulation conditions are shown in Table 8.

Table 8. Simulation conditions.

\begin{tabular}{ll}
\hline Items & Values \\
\hline Start time (UTC) & Jan. 1st 2016 00:00:00 \\
End time (UTC) & Jan. 1st 2016 00:30:00 \\
Target location & $75.0097^{\circ} \mathrm{S}, 10.25444^{\circ} \mathrm{E}$ \\
Closest approach time (UTC) & Jan. 1st 2016 00:15:00 \\
Initial attitude quaternion & {$[0,0,0,1]$} \\
Initial angular velocity & {$[0.2,0.2,0.2]^{\circ} / \mathrm{s}$} \\
Initial RW speed & {$[0,0,0,0] \mathrm{rpm}$} \\
\hline
\end{tabular}

\subsection{Attitude control procedure}

To execute ground-target tracking, it requires a special attitude control procedure since we cannot use STTs during the maneuver. RISESAT keeps Local Vertical, Local Horizontal (LVLH) attitude as a nominal mode. At 00:06:00, RISESAT halts pitch-axis rotation and switch to inertia fixed attitude control mode. This mode enables to determine more precise attitude using STT-IIR filter. The attitude control procedure is shown in Table 9.

Table 9. The Attitude control procedure.

\begin{tabular}{ll}
\hline Time (UTC) & Control Mode \\
\hline 00:00:00-00:03:00 & Tumbling (no control) \\
00:03:00-00:06:00 & LVLH attitude \\
00:06:00-00:10:30 & Inertia fixed (with STT) \\
00:10:30-00:20:00 & Ground-target tracking \\
00:20:00-00:30:00 & LVLH attitude \\
\hline
\end{tabular}

\subsection{Evaluation results}

CG views during ground-target tracking are shown in Fig. 12. RISESAT is controlled to keep orientation of on-board camera images to constant direction.

\section{Ground-target tracking error}

Ground-target tracking error which is an angle between desired boresight vector and satellite $z$-axis is shown in Fig. 13. A zone painted by gray indicate the period under tracking control. For approximately 3 minutes from 00:14:48 to 00:17:40, tracking error goes below required control accuracy $0.1^{\circ}$. Especially, error angle was $0.054^{\circ}$ when the satellite fly over the ground target at 00:15:00.

\section{Attitude stability}

Attitude Stability to desired boresight vector is shown in Fig. 14. To avoid blurring during exposure, $\pm 0.008 \% \mathrm{~s}$ attitude stability is required. From Fig. 14, we can find that the RISESAT satisfy the observation requirement.

\section{Attitude determination error}

Unlike prior works, we evaluated the attitude control system under influence of sensor noise, computation delay and

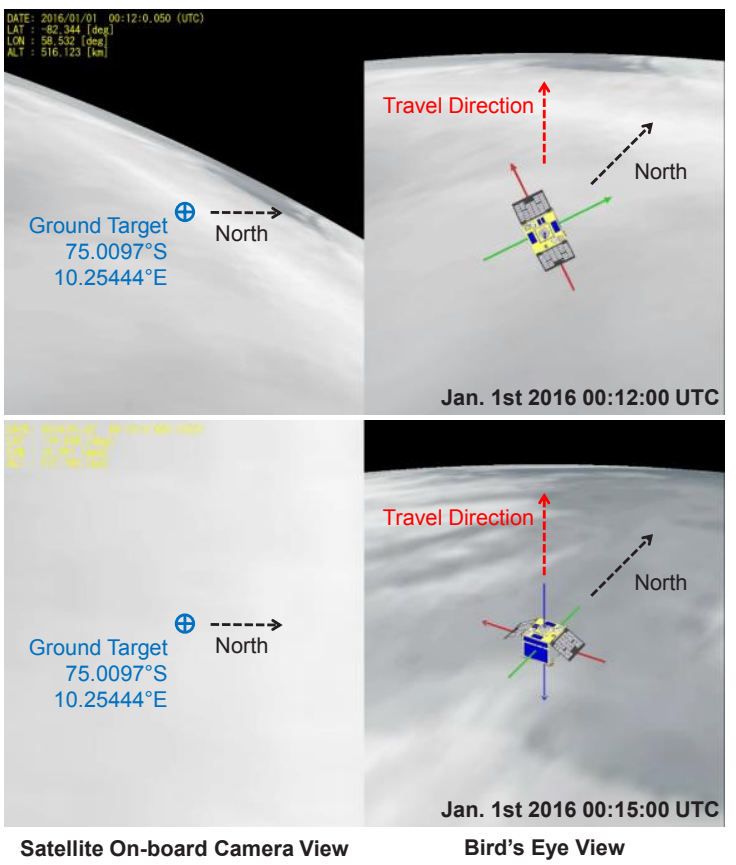

Fig. 12. CG Views during ground-target tracking control.

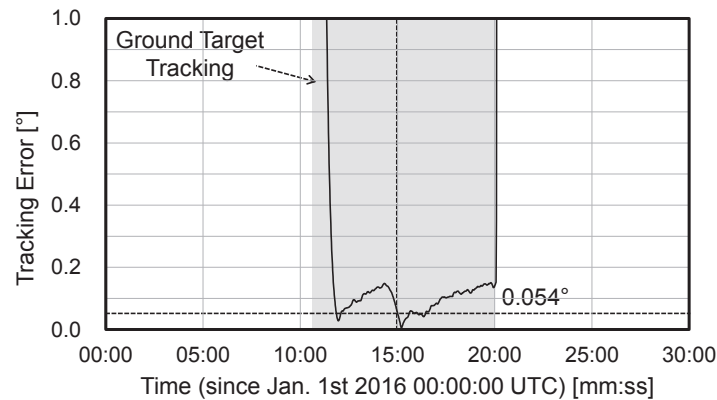

Fig. 13. Tracking error.

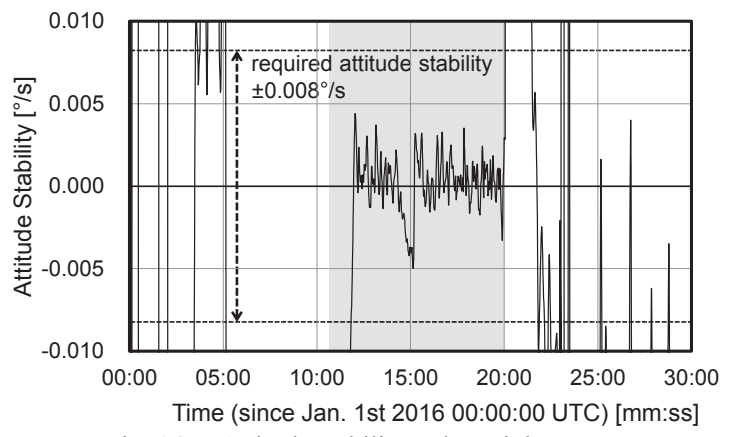

Fig. 14. Attitude stability to boresight vector.

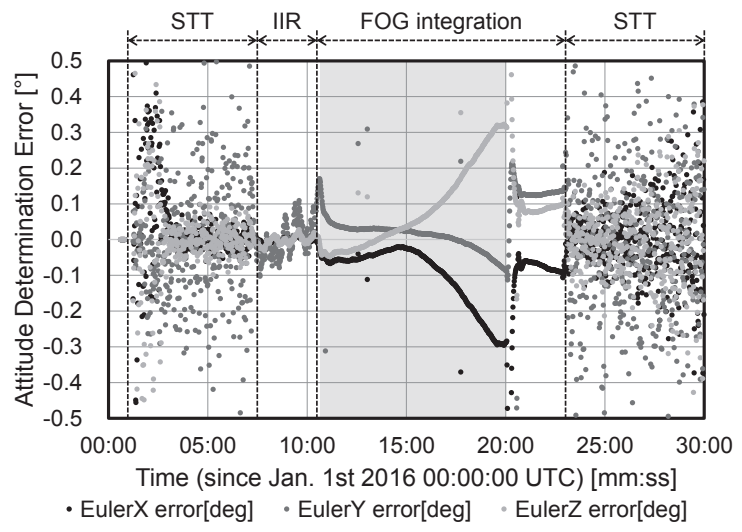

Fig. 15. Attitude determination error. 
numerical error. From Fig. 15, we can find that IIR filter lower random noise of attitude determined by a STT. After the attitude determination mode was changed to FOG integration, determination error increase as time proceeds. This phenomenon is caused by angular velocity measurement error such as random walk and numerical integration error. However, error at 00:15:00 satisfy the control requirements.

\section{Conclusions}

This paper presents a ground-target tracking algorithm for multi-spectral snapshot observation. Characteristics of this algorithm is it can keep orientation of ground-target images to a constant direction which the satellite operator requires. Owing to this, synthesis of images taken by different bands becomes easier. To carry out multi-spectral snapshot observation by RISESAT with HPT, $0.1^{\circ}$ attitude control accuracy and $0.008 \%$ s stability is indispensable.

Desired target attitude can be derived by calculating boresight vector from a satellite to ground target, and using orthogonal projection of north direction vector at the target area to the plane which is perpendicular to the boresight vector. Desired angular velocity can be obtained from time derivative of desired attitude. To determine desired control torque, we apply quaternion-based rate/attitude tracking control algorithms with desired angular acceleration feedforward proposed by Weiss.

The control algorithms and attitude control system were evaluated by hardware-in-the-loop simulator MEVI $\mu \mathrm{S}$ developed by SRL. The simulation results show that both control accuracy and stability satisfy mission requirements under influence of sensor noise, communication error and computation time limits.

The algorithm proposed in this paper will contribute to enable $5 \mathrm{~m}$ GSD and 680 bands multi-spectral observation by RISESAT and future microsatellites.

\section{Acknowledgments}

This research is granted by Tohoku University Division for Interdisciplinary Advanced Research and Education. RISESAT project is supported by Innovative Satellite Technology Demonstration Program of JAXA.

\section{References}

1) D'Angelo, P., Kuschk, G. and Reinartz, P.: Evaluation of Skybox Video and Still Image products, International Archives of the Photogrammetry, Remote Sensing and Spatial Information Sciences, XL-1 (2014), pp.95-99.

2) Aoyanagi, Y., Iwasaki, A., Nakasuka, S., Ebinuma, T., Kimura, S., and Narumi, T., Results of Hodoyoshi-3 and -4 Earth Observation Missions, Trans. JSASS Aerospace Tech. Japan, 14 (2016), pp. Pn_15 - Pn_22.

3) Kurihara, J. and Takahashi, Y.: High-Spatial Resolution MultiSpectral Imaging with the HPT on the RISESAT Micro-Satellite, Proceedings of the 60th Space Science and Technology Conference, Hakodate, 2016 (in Japanese).

4) Takahashi, Y., Kurihara, J. and Fukuhara, T.: Advanced RemoteSensing Sensors for Micro-satellite and Their Applications, IEICE Technical Report, 111 (2011), (in Japanese).

5) Kramer, H. J.: Observation of the Earth and Its Environment, Springer, Berlin, 1996.

6) Kuwahara, T., Yoshida, K., Sakamoto, Y.: Development Status of Micro-satellite RISESAT, Proceedings of the 60th Space Science and Technology Conference, Hakodate, 2016 (in Japanese).

7) Goeree, B. B. and Shucker, B.: Geometric Attitude Control of a Small Satellite for Ground Tracking Maneuvers, Proceedings of the 13th Annual AIAA/Utah State University Conference on Small Satellites, Utah, 1999.

8) Chen, X., Steyn, W. H. and Hashida Y.: Ground Target Tracking Control of Earth-Pointing Satellites, AIAA Paper 2000-4547, 2000.

9) Kondo, Y., Ohkami, Y. and Taniwaki, S.: Study of Ground Point Tracking of Earth Observation Satellites, Journal of the JSASS, 52 (2004), pp.23-29.

10) Sakamoto, Y., Kuwahara, T., Yoshida, K.: Development and Operation Progress of Philippines' Microsatellite DIWATA-1, Proceedings of the 60th Space Science and Technology Conference, Hakodate, 2016 (in Japanese).

11) Vallado, D. A.: Fundamentals of Astrodynamics and Applications 4th edition, Microcosm Press, Hawthorne, 2013.

12) Montenbruck, O. and Gill, E.: Satellite Orbits, Springer, Berlin, 2000.

13) Weiss, H.: Quaternion-Based Rate/Attitude Tracking System with Application to Gimbal Attitude, Journal of Guidance, Control and Dynamics, 16 (1993), pp.609-616.

14) Wie, B. and Weiss, H.: Quaternion Feedback Regulator for Spacecraft Eigenaxis Rotations, Journal of Guidance, Control and Dynamics, 12 (1989), pp.375-380.

15) Petit, G., Luzum, B.: IERS Conventions 2010, IERS Technical Note No.36, 2010.

16) IERS Bulletin A, https://www.iers.org/IERS/EN/Publications/ Bulletins/bulletins.html (accessed April 1, 2017).

17) Iwata, T., Hoshino, H. and Yoshizawa, T.: Precision Attitude Determination for the Advanced Land Observing Satellite (ALOS): Design, Verification, and On-Orbit Calibration, AIAA Paper 20076817, 2007.

18) Markley, F. L., Cheng, Y., Crassidis, J. L. and Oshman, Y.: Averaging Quaternions, Journal of Guidance, Control, and Dynamics, 30 (2007), pp. 1193-1197.

19) Tomioka, Y., Yoshida, K., Sakamoto, Y., Kuwahara, T., Fukuda, K., Sugimura, N., Fukuyama, M. and Shibuya, Y.: Establish the Environment to Support Cost-effective and Rapid Development of Micro-satellites, Proceedings of the International Astronautical Congress, Naples, 2012, pp.8470-8477.

20) Space-Track, https://www.space-track.org/ (accessed April 1, 2017). 